COMECHINGONIA. Revista de Arqueología. Vol. 19, n 1. Primer semestre de 2015. ISSN: 0326-7911

\title{
RESTOS ARQUEOBOTÁNICOS DEL SITIO RINCÓN CHICO 1. UNA APROXIMACIÓN A LOS POSIBLES ESCENARIOS DE PROCESAMIENTO, USO Y CONSUMO.
}

\section{ARCHAEOBOTANICAL REMAINS FROM RINCÓN CHICO 1 SITE. AN APPROACH TO POSSIBLE PROCCESING, USE, AND CONSUMPTION SCENARIOS.}

\author{
Natalia Petrucci ${ }^{1}$ y Myriam Tarragó ${ }^{2}$ \\ ${ }^{1}$ Museo Etnográfico, FFyL-UBA; Moreno 350 (CP: 1091), CABA, Argentina. E-mail: \\ nataliapetrucci@hotmail.com; \\ ${ }^{2}$ Museo Etnográfico, FFyL-UBA-CONICET; Moreno 350 (CP: 1091), CABA, Argentina. E-mail: \\ tarragomyriam@gmail.com
}

Presentado: 10/12/2014 - Aceptado: 14/4/2015

\section{Resumen}

La excavación por muestreo estratificado en el asentamiento prehispánico de Rincón Chico -Sitio 1-, Departamento de Santa María, Provincia de Catamarca, perseguía entre sus objetivos, obtener información no sesgada sobre el conjunto de estructuras arquitectónicas, según forma y tamaño, y recuperar restos arqueobotánicos. Con tales propósitos, en las campañas de 1992 y 1995, se implementó el uso de la técnica de flotación por medios manuales. El presente trabajo presenta el análisis del conjunto de restos botánicos carbonizados que proceden de pisos de ocupación. De estos restos, se seleccionaron aquellos relacionados con el consumo alimenticio. Se dan los fundamentos del método, el modo en que se implementó y se discuten los resultados obtenidos.

Palabras clave: Argentina, Catamarca, Rincón Chico, restos botánicos

\footnotetext{
Abstract

The excavation using a stratified sampling strategy in the prehispanic settlement of Rincón Chico Site 1, Department of Santa María, Province of Catamarca, pursued, among its goals, to obtain unbiased information about the set of architectural structures, according to shape and size, and to recover archaeobotanical remains. With such purposes, in the campaigns of 1992 and 1995, the use of the manual flotation technique was used. This paper presents the analysis of the set of
} 
charred botanical remains coming from occupational floors. From the recovered botanical remains, those related to food consumption were selected. The fundamentals of the method and the way it was implemented are presented and results are discussed.

Key-words: Argentine, Catamarca, Rincón Chico, botanical remains

\section{Introducción}

La excavación por muestreo estratificado en el asentamiento prehispánico del Sitio 1 de Rincón Chico, Departamento de Santa María, Provincia de Catamarca, perseguía entre sus objetivos, obtener información no sesgada, sobre el conjunto de estructuras arquitectónicas según forma y tamaño, y recuperar restos arqueobotánicos. Como se ha indicado en otros trabajos (Tarragó 2011), el Sitio 1 corresponde a un poblado emplazado sobre la cima, las laderas y el pie de un cerro de la Sierra del Cajón, ubicado cronológicamente entre los siglos XI y XVI de nuestra era. Se trata de un conglomerado arquitectónico que combina diversos grupos de estructuras con muros de piedra que ha sido definido como un tipo de "pukara" del valle de Yocavil (Tarragó 2011).

Con tal propósito, en las campañas de 1992 y 1995 se implementó el uso de la técnica de flotación por medios manuales. Esta técnica, aplicada a sedimentos de sitios arqueológicos, tiene como finalidad recuperar aquellos elementos que, por su reducido tamaño, usualmente no son detectados durante el proceso de excavación ni son recuperados, sin deterioro, en la zaranda de malla fina. Los restos pueden ser de naturaleza vegetal, animal o mineral. En este caso se buscaba la recuperación específica de restos arqueobotánicos que son tan valiosos para la reconstrucción de la historia ambiental, la acción antrópica sobre los paisajes y fundamentalmente, el aprovechamiento de los recursos del medio ambiente, tanto silvestres como cultivados. De ellos se puede derivar información sobre el consumo alimenticio de determinadas plantas así como diversos usos domésticos de los materiales vegetales en techados, cestería, trenzados y sogas, entre otros.

El presente trabajo retoma los estudios y las determinaciones realizadas en la década de los noventa (Arriaga et al. 1994) ampliando el análisis al conjunto de restos botánicos carbonizados obtenidos por dicho método y procedentes de pisos de ocupación. Se dan los fundamentos del método, el modo en que se implementó y se discuten los resultados obtenidos ${ }^{1}$.

\section{Excavaciones por muestreo en Rincón Chico 1}

La localidad arqueológica de Rincón Chico, localizada en la comuna de Lampacito, comprende alrededor de 500 hectáreas entre agrupamientos constructivos, áreas de producción y tránsito, incluyendo localizaciones de recursos minerales, bióticos e 
hídricos. Atendiendo a la continuidad espacial, cada uno de los agrupamientos constructivos dentro de la localidad arqueológica, fue denominado "sitio" y se les concedió un número. La prospección pedestre sistemática posibilitó la detección de 37 lugares con restos de arquitectura visible en superficie.

El presente trabajo se relaciona específicamente con el Sitio 1 (RCh1), que se emplaza encima del espolón de rocas metamórficas de origen precámbrico, las laderas en forma de farallones y la parte apical del abanico aluvial (Ruiz Huidobro 1972). La superficie relevada abarcó una superficie total de $395.704 \mathrm{~m}^{2}$ (39,6 ha). El punto topográfico más bajo se ubica a $1956.04 \mathrm{msnm}$, en tanto que el punto más alto se encuentra a 2164.26 msnm. Se estableció, por lo tanto, una altura real del cerro de 208.22 metros.

El área del conglomerado fue dividida, para su mejor observación, en XIII sectores en consideración a aspectos topográficos y de escurrimiento (Figura 1). Cada estructura fue dibujada en escala 1:100 y descripta in situ con sus particularidades constructivas y su estado de conservación. La unidad básica de edificación está dada por la combinación de recintos rectangulares u ovales, intercomunicados. En las zonas escarpadas, la unidad suele estar dada por un recinto rectangular que desemboca en una explanada o plataforma, a modo de "balcones". En pocos casos se asocia además un recinto circular (Tarragó 1987, 1995).

Las grandes dimensiones y la complejidad arquitectónica del poblado-Pukara de RCh1 nos llevaron a plantear una investigación que permitiera abarcar el conjunto a través de una técnica de muestreo en la cual estuvieran representadas las distintas variables de modalidad arquitectónica y de emplazamiento en los distintos sectores del asentamiento. Los objetivos básicos que nos guiaron fueron: (1) obtener información cronológica a fin de establecer el lapso temporal y la secuencia de crecimiento del poblado; (2) registrar la variabilidad intersectorial en la distribución de restos culturales y técnicas constructivas; (3) tomar conocimiento de la potencia de los sedimentos, la estratigrafía de los depósitos en los distintos sectores y las perturbaciones postdeposicionales; (4) recopilar información para seleccionar lugares del sitio para ser sometidos a excavaciones de mayor envergadura; (5) recuperar por medio de la flotación, micro-especímenes que, por su reducido tamaño, usualmente no son detectados durante el proceso de excavación ni son recuperados sin deterioro de la zaranda.

Para tal fin se encaró el diseño de un programa de muestreo que permitiera atender satisfactoriamente a estos objetivos, se adecuara a los recursos y tiempo disponibles, fuera de aplicación práctica y redujera al mínimo la alteración de los restos arqueológicos. Por una parte, las evidencias arquitectónicas de superficie y su disposición en el espacio mostraban una variabilidad que aconsejaban establecer una jerarquización interna en el universo muestral. Por otro lado era importante recuperar de las unidades de muestreo información de similar valor comparativo, dejando lugar además, para la aparición de evidencias que no estuvieran incluidas en las expectativas previas, a través de atender puntos del sitio que, en una excavación estrictamente dirigida, probablemente no hubieran sido considerados (Lennstrom y Hastorf 1995; Mueller 1979). 


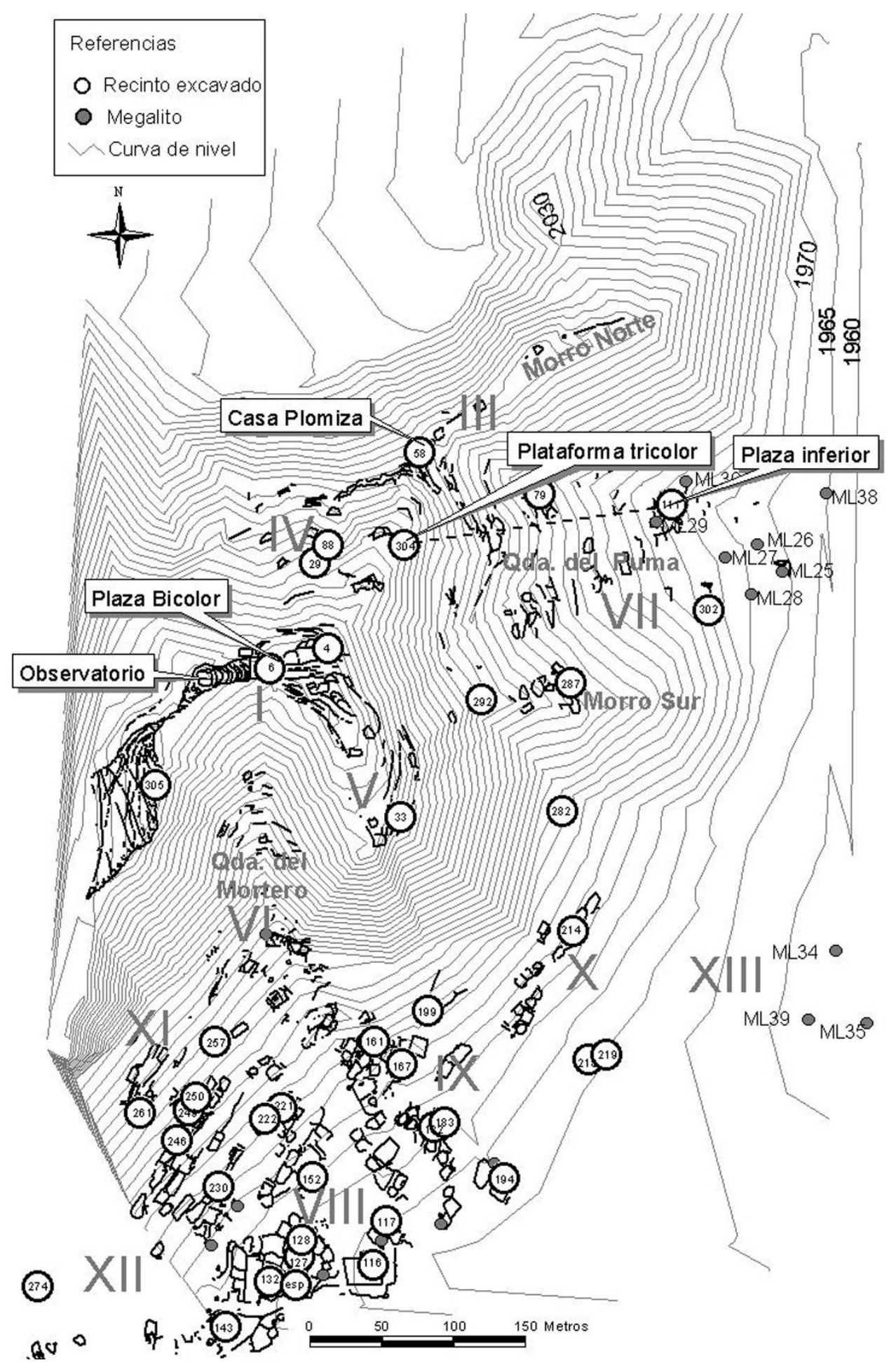

Figura 1. Plano de Rincón Chico 1.Sectorización y recintos excavados (según Tarragó 2011, Fig. 4). 
Para reducir la heterogeneidad del universo, el sitio fue dividido en cinco estratos que incluía un número similar de estructuras, sobreimpuestos a la sectorización referida líneas atrás. El Estrato 1 incluyó los sectores I, II, III, IV y V de la cumbre, talud norte y parapetos del talud occidental; el Estrato 2, el sector VII de la Quebrada del Puma; el Estrato 3, los sectores VIII - XII del poblado bajo sur; el Estrato 4, los sectores IX y X del poblado norte y el Estrato 5, los sectores XI y VI del poblado alto meridional y el anfiteatro de escurrimiento del arroyo de Los Morteritos (Tarragó et al. 1992). El sector XIII, zona de megalitos con morteros, fue establecido con posterioridad y no comprende recintos pircados. Por estos dos motivos no se contempló en el diseño.

Asimismo, a los fines de lograr niveles operativos adecuados, en esa primera etapa de excavación en RCh1 se optó por dejar de lado a los espacios aledaños a los recintos y a las estructuras denominadas Plataformas, limitándose el ámbito de muestreo a las superficies intramuros. Sobre la base de 253 recintos registrados, se seleccionó una muestra de 34 estructuras $(13.43 \%$ ) por muestreo al azar, en la que estuvieran representados los cinco estratos según la forma (rectangular y circular) y por tamaño: pequeño (hasta $\left.30 \mathrm{~m}^{2}\right)$, mediano $\left(31\right.$ a $\left.60 \mathrm{~m}^{2}\right)$, grande $\left(61\right.$ a $\left.199 \mathrm{~m}^{2}\right)$ y muy grande $\left(200 \mathrm{~m}^{2}\right.$ y más). El objetivo era hacer una excavación exploratoria no dirigida en el interior de los mismos.

La unidad de intervención, seleccionada también por muestreo al azar simple, fue de $1 \mathrm{~m}^{2}$ cubriéndose 21 estructuras durante la campaña de 1992 y las 13 restantes en 1995. En varios de los casos, debido al derrumbe de los muros, se hizo necesario ampliar la excavación a $2 \mathrm{~m}^{2} \mathrm{o}$ a una extensión mayor. Este cubrimiento posibilitó tomar muestras de un volumen constante, para ser procesadas por la técnica de flotación. Se flotó 1 litro de sedimento extraído al azar, por cada uno de los niveles excavados, en cada cuadrícula del muestreo. Por este procedimiento se recuperaron pelos, fibras, semillas, hojas, raicillas, guano, espículas de carbón, conchillas, microlascas de obsidiana, cuentas de collar, entre otros materiales (Arriaga et al. 1994). En casi todos los casos, se determinó el piso de ocupación, a una profundidad variable entre 0.2 a $0.8 \mathrm{~m}$ bajo superficie, y los rasgos asociados al mismo como estructuras de combustión, restos de fauna, industria lítica y fragmentos de cerámica. En los recintos en que se recolectó una buena muestra de carbón vegetal, se efectuaron fechados por radiocarbono (Figura 1).

Hasta el momento contamos con 14 dataciones para RCh1 (sobre un total de 40 para toda la localidad) que nos ofrecen un panorama bastante definido en relación a la amplitud o rango temporal de ocupación, pero fundamentalmente, de aquellos momentos de mayor intensidad de uso del poblado, entre los siglos XIII y XVI d.C. (Tarragó 2011: 51, Tabla 2). 
En síntesis, fueron detectadas interesantes áreas de actividad, se confirmó la excelencia de detalles constructivos ya observados en superficie y en cuanto a la formación de sitio y se registraron las condiciones estratigráficas locales. Los ecofactos y restos recuperados de la flotación demostraron la importancia de la aplicación del método de muestreo y de la técnica de flotación. Debe destacarse que no existían antecedentes de la aplicación de esta técnica en el valle de Santa María con anterioridad a esta experiencia.

\section{El ambiente donde se emplaza el sitio Rincón Chico 1}

El Sitio 1 de Rincón Chico, como ya señalamos, se dispone sobre un espolón rocoso de la Sierra del Cajón y la cúspide de un conoide de deyección que se extiende al pie. El resto de los 36 sitios se distribuyen sobre este último. Los conos o abanicos aluviales no constituyen un ambiente uniforme, pues en ellos se puede distinguir una porción apical, de materiales más gruesos y pendientes más acentuadas, próxima al bloque montañoso y una porción distal, formada por materiales cada vez más finos, en la cual la humedad va en aumento y permite la existencia de vegetación de mayor porte (Grondona 1978: 121).

El clima en Santa María es seco y cálido-templado. Las lluvias no alcanzan los 200 $\mathrm{mm}$ anuales lo cual hace que toda la actividad agrícola se base en el regadío y que el río homónimo y sus afluentes de régimen permanente, tengan su alimentación en la neviza que se forma en las altas cumbres por influjo de los vientos alisios.

Los recursos vegetales del espacio circundante se relacionan por una parte con la provincia del Monte, que incluye la típica formación de bosquecillos en galería a lo largo de la vaguada del río de algarrobo (Prosopis alba, P. nigra) y chañar (Geoffroea decorticans). El carbón de madera de algarrobo parece haber sido un recurso crítico en la producción metalúrgica prehispánica en Yocavil (González 1995). La formación de matorral espinoso y de cactáceas vinculado a la provincia de la Prepuna como los "cardones" (Trichocereus sp.) y las bromeliáceas (Abromeitiella sp.), apropiadas para la extracción de madera y fibras respectivamente, se desarrollan en las laderas de los cerros y en el plano inclinado de los conoides. Un primer análisis del herbario obtenido de un transepto en el conoide de Rincón Chico (Cabrera 1990) y la identificación de microespecímenes de flotación en el recinto 128 del RCh1 muestran correspondencia con esas formaciones de vegetación (Arriaga et al. 1994). Por otro lado, hay que destacar para fines comestibles, el consumo del fruto del algarrobo, la preparación de harinas y de bebidas fermentadas. Del mismo modo, el fruto del cardón pasacana (Echinopsis atacamensis var. pasacana) ha sido muy apetecido para consumo fresco como para producir bebidas fermentadas. 


\section{Materiales y métodos}

En total se flotaron 156.25 litros de sedimento, de los cuales 106.25 litros estaban asociados al piso de ocupación o en los casos que no se pudo determinar el piso, se consideraron los últimos niveles por encima del estéril ${ }^{2}$. Merced a la diferencia de peso específico y de densidad de los elementos se obtuvo una fracción liviana y una fracción pesada. En el laboratorio ambas fracciones fueron íntegramente observadas con microscopio estereoscópico, de esta manera se dividieron los restos antracológicos de los carporrestos. De los carporrestos recuperados se seleccionaron aquellos relacionados al consumo alimenticio. La identificación taxonómica se realizó empleando microscopio estereoscópico y óptico con luz incidente. Con fines comparativos se confeccionó una colección de referencia (herbario de N. Petrucci) de la zona en estudio. Los vouchers se hallan depositados en el herbario del Laboratorio de Etnobotánica y Botánica Aplicada (LEBA) de la Facultad de Ciencias Naturales y Museo (FCNyM-UNLP). Se utilizó material bibliográfico de referencia como las floras argentinas (Cabrera 1976), Catálogo de las Plantas Vasculares del Conosur, textos sobre caracteres diagnósticos y claves de determinación (Winton y Winton 1932, 1935), y se consultó la colección científica de plantas utilitarias del Laboratorio de Etnobotánica y Botánica Aplicada (FCNyMUNLP).

Una vez analizada e identificada la muestra total de carporrestos de RCh1 ( $n=375)$, se efectuó una segunda separación considerándose solamente aquellos restos que se encontraron en los niveles ocupacionales del sitio. El clima de tipo semidesértico no favorece la preservación de materiales orgánicos, de manera que para asegurar que los restos arqueobotánicos analizados correspondiesen a las ocupaciones pretéritas del sitio, se consideraron sólo los que estaban en estado carbonizado $(n=214)$. Luego de la identificación y discriminación por especie, los restos fueron contados. Se elaboraron tablas con todos los datos obtenidos. Para el análisis cuantitativo se utilizaron cantidades absolutas y porcentuales de cada taxón para estimar la representación de cada uno en el sitio. Las cantidades absolutas se expresaron como el número entero de restos (ya sea que estos estuvieran enteros o fragmentados) de un determinado taxón por unidad de procedencia.

Pero debido a que esta cantidad así como la frecuencia absoluta no tiene en cuenta los problemas de preservación diferencial de los restos arqueobotánicos (Popper 1988), no se utilizaron con fines comparativos entre muestras. Luego se estimaron los valores de densidad en cada estrato, expresada como cantidad de restos carbonizados por litro de sedimento flotado (n/litro) (Miller 1988: 73), esto con el objeto de poder comparar muestras de diferentes volúmenes y cantidades originales (Leenstrom y Hastorf 1992, 1995). 


\section{Resultados}

Del total de carporrestos carbonizados recuperados $(\mathrm{n}=214)$, se pudieron identificar el 92.5\%, determinandose los siguientes taxa: Zea mays (granos y cúpula/marlo) (Figura 2), Trichocereus sp. (semilla), Opuntia sp. (semilla) (Figura 3), Portulaca sp. (semilla), Prosopis sp. (endocarpo y semilla) (Figura 3), Poligonum sp. (semilla) y Chenopodium sp. (semilla).

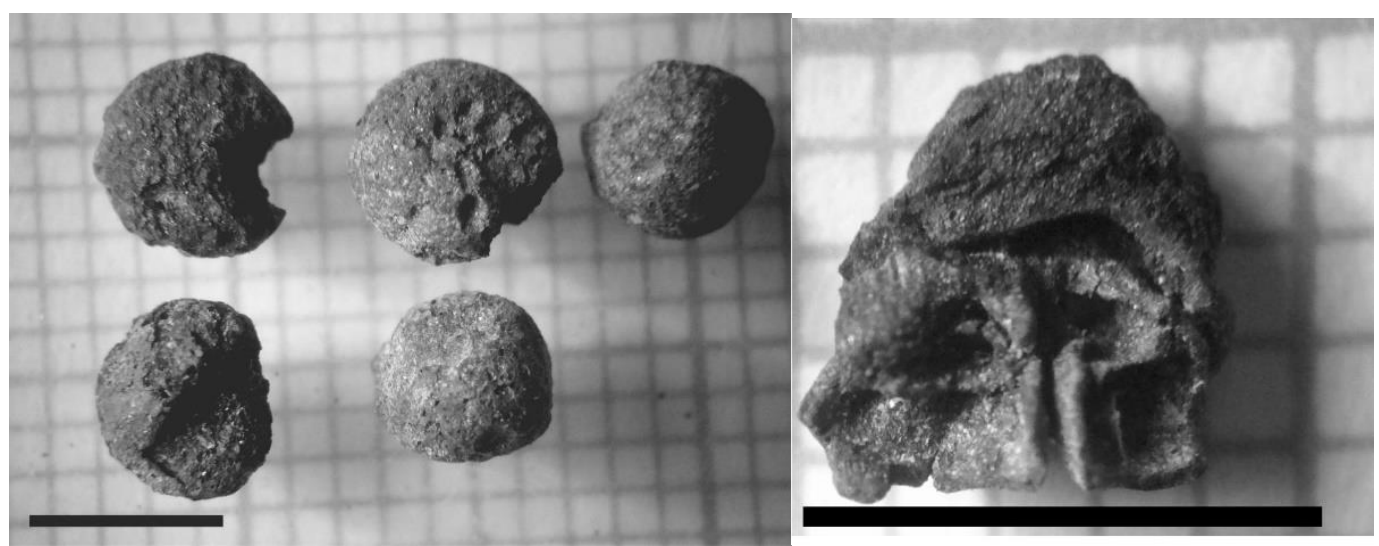

Figura 2. Restos de granos (izquierda) y fragmento de marlo (derecha) de Zea mays. Escala $=5 \mathrm{~mm}$.

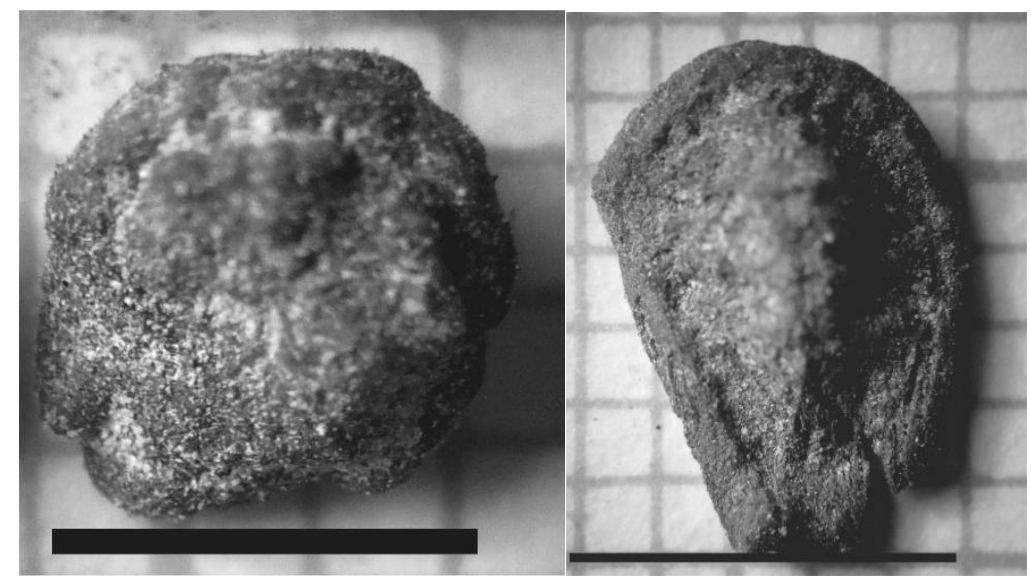

Figura 3. Semilla de Opuntia sp. (izquierda), escala=3mm. Endocarpo Prosopis sp. (derecha), escala $=5 \mathrm{~mm}$.

Las cantidades absolutas y porcentuales de los macrorrestos recuperados por flotación en la totalidad del sitio Rincón Chico 1 se resumen en la Figura 4. La especie más abundante es Zea mays, seguida por Prosopis sp., Trichocereus sp. y Opuntia sp.

Las densidades promedios en los distintos estratos mostraron que los mayores valores de restos arqueobotánicos derivaron del E3, que corresponde al poblado bajo Sur, mientras que los E1, E4 y E5 presentaron valores intermedios y el E2 el menor (Figura 5). 


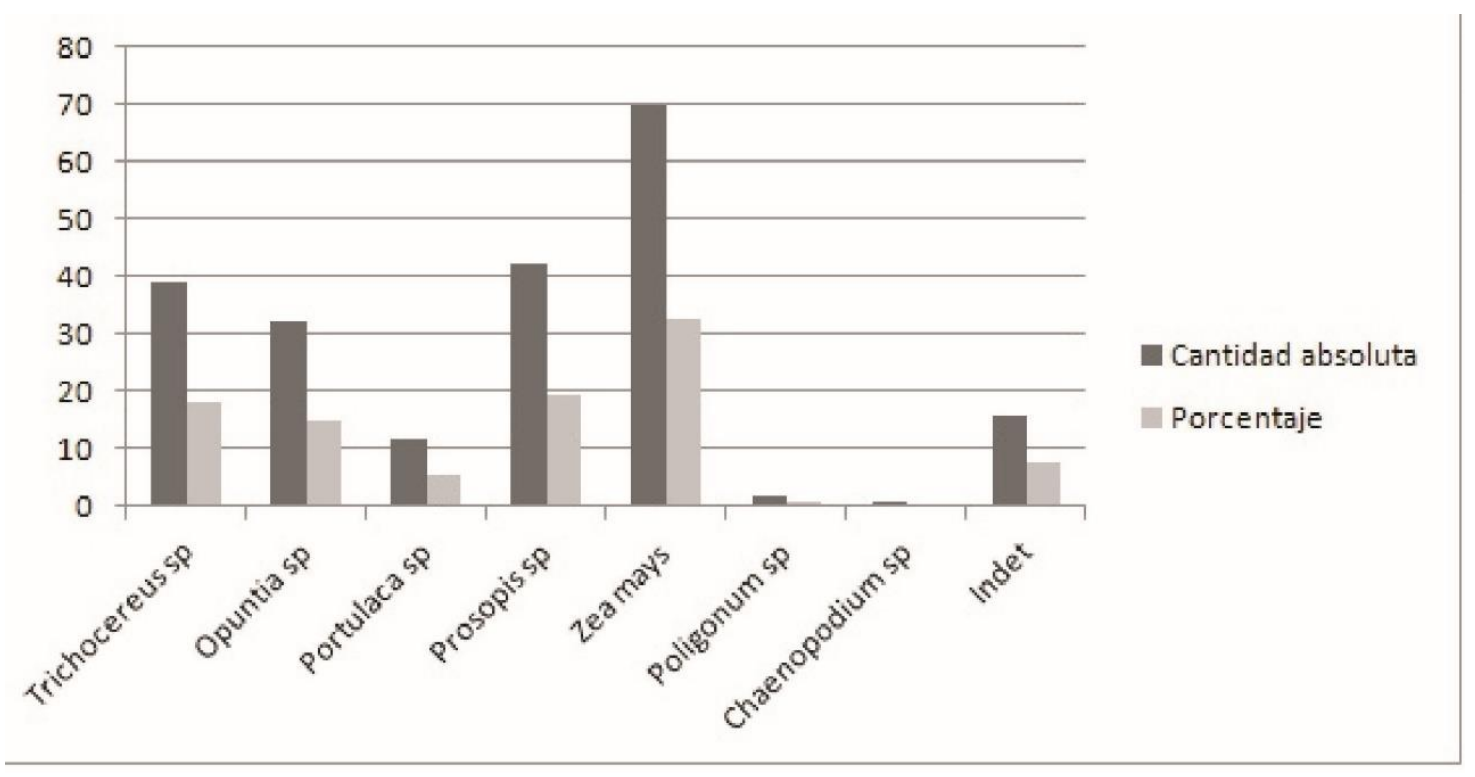

Figura 4. Cantidades absolutas y porcentuales de los macrorrestos recuperados.

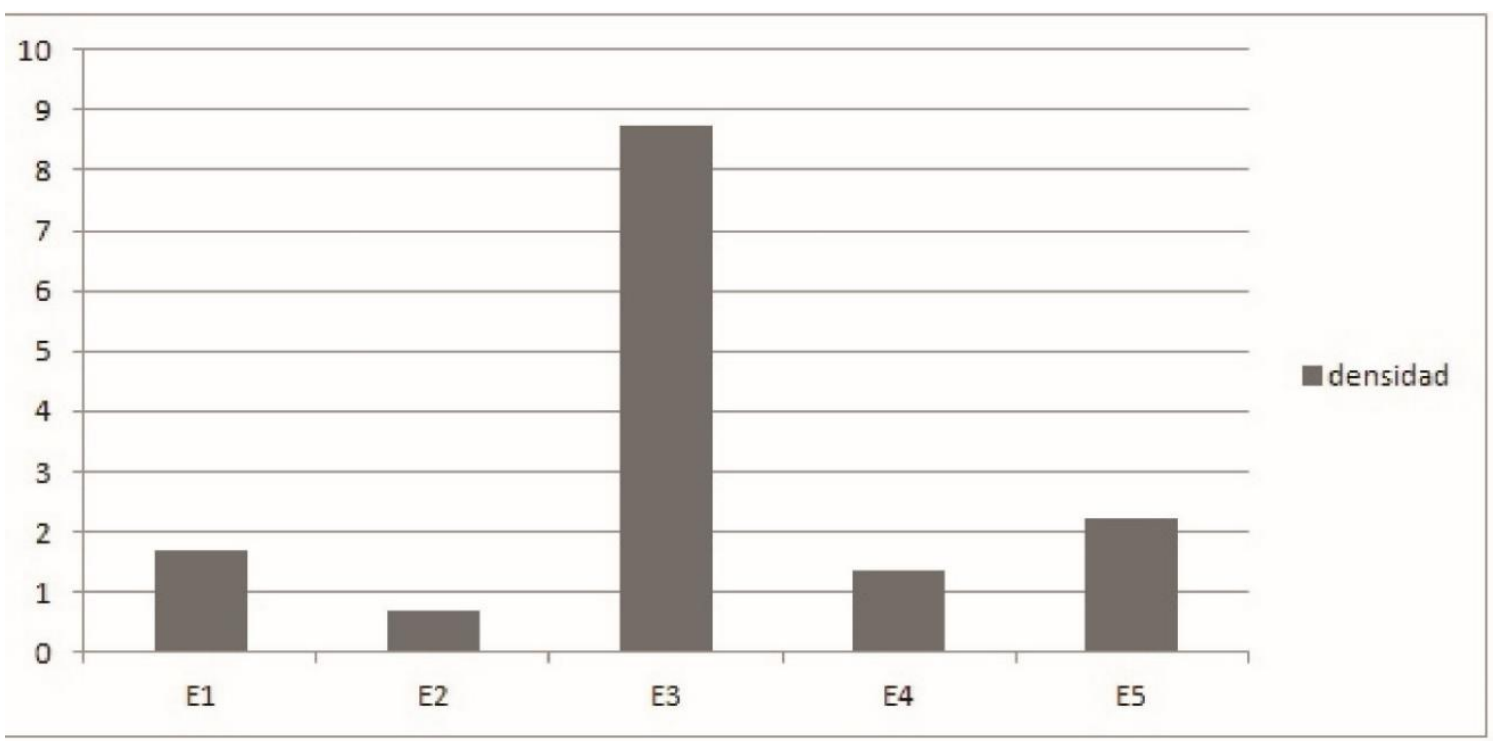

Figura 5. Densidad promedio de taxa vegetales recuperados en cada estrato.

El porcentaje de contribución de los taxa en cada estrato mostró que Prosopis sp. representa aproximadamente el 75\% de los restos recuperados en el estrato 3. En el E1 y el E5 se observa una situación parecida, donde Zea mays, Trichocereus sp. y Opuntia sp. están bien representados y con similares proporciones, aunque los porcentajes de restos de Zea mays son más altos en el E5 (52.7\%). Por otro lado Zea mays (36.4\%), Prosopis sp. $(31.8 \%)$ y Opuntia sp. $(27.3 \%)$ son los que mayores proporciones presentaron en el E4. Por último, Trichocereus sp. es el único taxón representado en el E2 (Figura 6). 


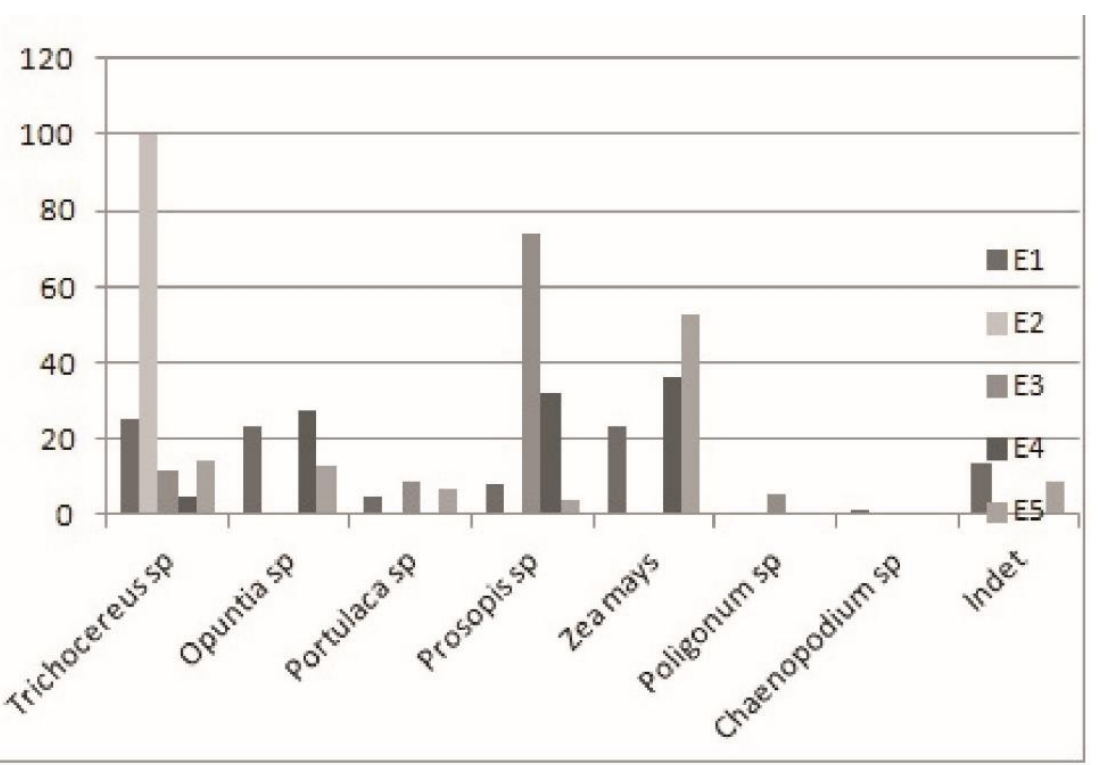

Figura 6. Porcentaje de contribución de los taxa en cada estrato.

El gráfico de la Figura 7 muestra las densidades de macrorrestos en los recintos excavados, pudiendo observarse que los mayores registros coinciden con los estratos que también presentaron los valores más altos de densidad.

Entre los macrorrestos recuperados correspondientes a Zea mays, los fragmentos de marlos (ya sean cúpulas o marlos) están más densamente concentrados que los granos en los E4 y E5, observándose la notable diferencia que sucede en el E1 donde los granos predominan sobre los marlos (Figura 8).

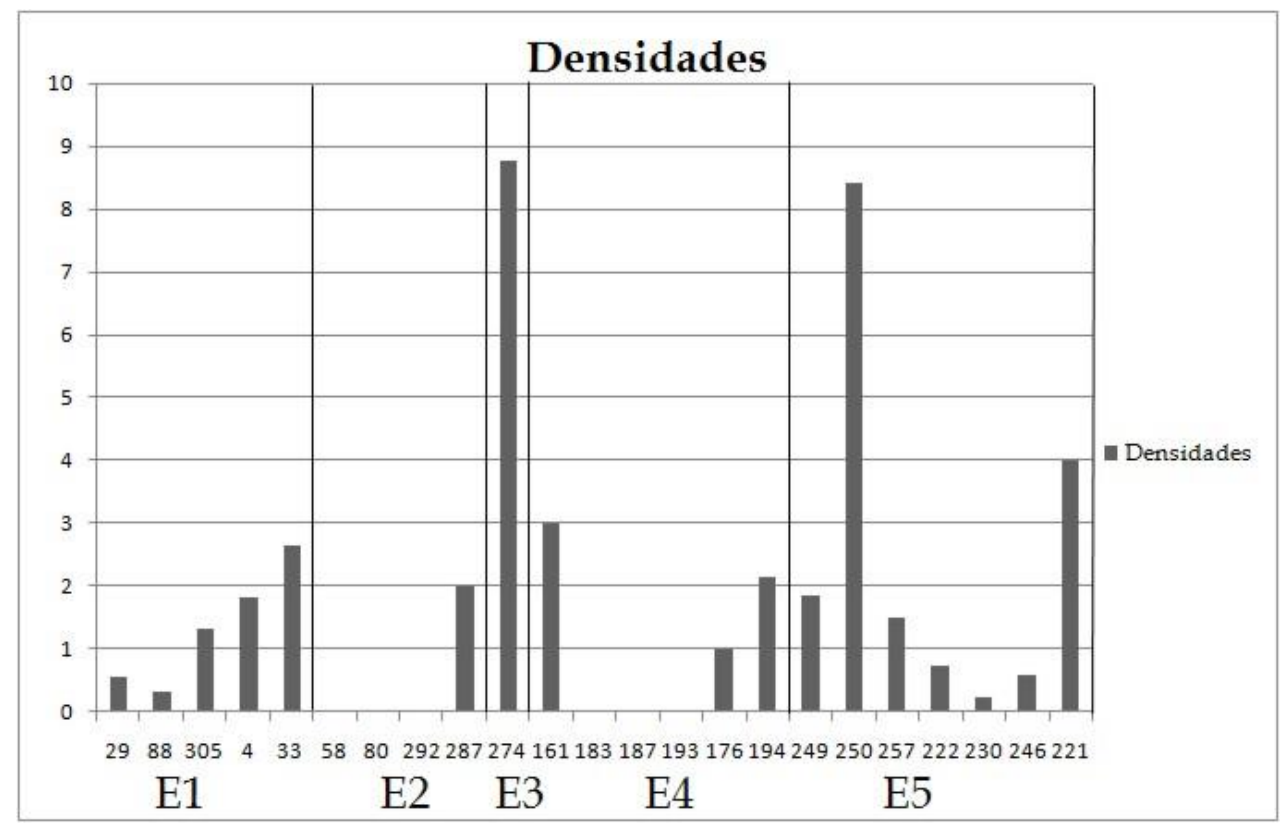

Figura 7. Densidad de restos por recinto excavado. 


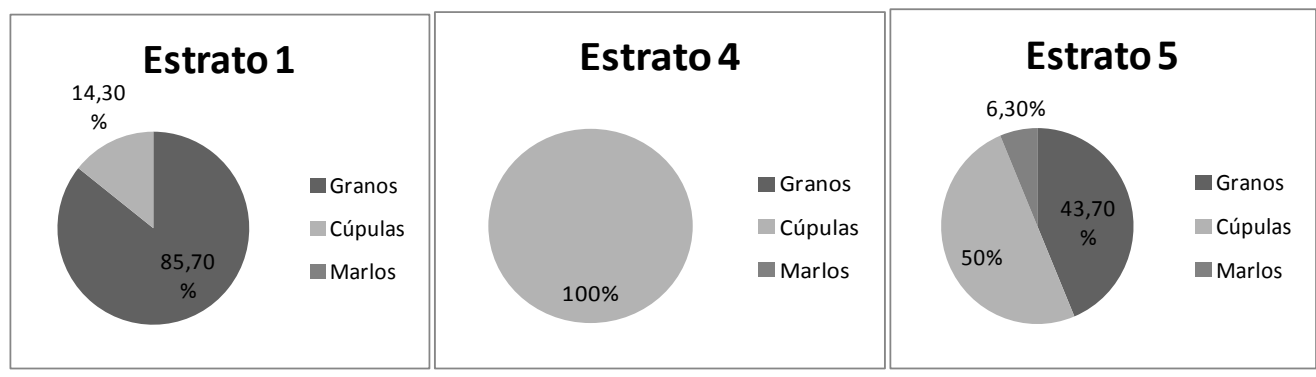

Figura 8. Porcentajes de restos de Zea mays recuperados en cada estrato.

\section{Discusión}

Preservación de los restos en la localidad Arqueológica de Rincón Chico

La evaluación de los resultados indica que, a pesar de la cantidad de litros flotados, la recuperación de material fue escasa. Es importante remarcar que la frecuencia o número de especímenes presentes en la muestra es el resultado de la acción de distintos factores de preservación diferencial, es decir, está sujeto tanto a pautas culturales como naturales que determinan, en última instancia, la conformación del conjunto (Popper 1988). La preservación diferencial local también incidió en la cantidad de hallazgos. Esto se evidenció durante la excavación ya que, en algunas cuadrículas, no se encontraron las áreas de fogón y en otras, solo se hallaron espículas de carbón. Dentro de las pautas culturales que pudieron afectar directamente la frecuencia de restos cabe mencionar que se ha constatado, en forma recurrente, la limpieza de los pisos de las viviendas de Rincón Chico. Esta cualidad sería un rasgo sintomático del grado de organización social y el cuidado del ámbito doméstico en las sociedades tardías de Yocavil. Dicha característica establece una notable diferencia de manejo del espacio habitado con respecto a los múltiples registros conocidos para los centros poblados contemporáneos de Quebrada de Humahuaca y Puna, donde la abundancia de residuos in situ es muy grande (Tarragó 2011: 55).

El pool de semillas asociadas al consumo, recuperadas de los distintos contextos excavados, pudieron ingresar en los recintos a través de distintas fuentes. Las semillas pudieron ser utilizadas para consumo o parte de descarte de un fruto comestible (ej. marlo de maíz para combustible), o las semillas ingresaron a los distintos recintos con las cosechas de cultivos o con las heces de los animales que pudieron utilizarse como combustible (Pearsall 1988). Los restos recolectados se encontraron todos en estado carbonizado, esto puede deberse a las distintas rutas que siguieron las diferentes partes (utilizables o no) de las plantas. Por otro lado, en los valles semiáridos como Santa María y Calchaquí, la conservación de restos orgánicos en estado seco solo se da en sitios bajo roca como cuevas, por ejemplo "Los Graneros" y la "Cueva del Puente de Diablo". En cambio, a cielo abierto solo se recuperan en general los materiales botánicos que sufrieron algún proceso de carbonización, sin llegar a la destrucción total (Lennstrom 1992: 2; Raffaele 2008: 289-290). Las partes no consumibles pudieron ser utilizadas como combustible o haber caído en los suelos y luego con la limpieza de los pisos, arrojadas a 
los fogones. Las partes utilizables pudieron constituir desechos de comidas, ejemplares descartados por daño o que, accidentalmente, hayan caído a los fogones durante la preparación de alimentos (Munson et al. 1971).

Las evidencias de marlos carbonizados en el montículo oriental del sitio 15 de Rincón Chico, analizadas por Raffaele (2008: 315), añade mayor complejidad al problema de la preservación al proponer el uso sistemático de marlos como combustible para el encendido inicial de las estructuras de combustión destinadas a la producción metalúrgica y alfarera estudiadas en dicho sitio. En un segundo momento del encendido se habría usado "carbón" de madera de algarrobo para levantar temperaturas mayores a 1000 grados C. (González 1995, 2010; Tarragó 2007). En este sentido, la información de restos vegetales desprendida del análisis del Recinto 128 indica la presencia de foliolos de Prosopis sp. y restos de leños carbonizados en el área de piso, entre 80-115 cm bajo superficie (Arriaga et al. 1994). Con estos datos se cierra el círculo de aprovechamiento y uso como alimento, combustible y materia prima dentro del asentamiento, de un recurso tan valorado por los pueblos originarios como fue el algarrobo.

Las especies recuperadas en el sitio Rincón Chico 1

La diversidad de vegetales recuperados en el sitio RCh1 es baja, aunque la mayoría coincide con taxa que en la actualidad son utilizados como alimentos por los habitantes de los Andes: maíz, algarrobo, cactáceas, como muestran muchos trabajos etnobotánicos en el área (Pochettino y Scattolin 1991). Dos especies aparecieron en el registro arqueobotánico: Portulaca sp. y Polygonum sp., que en la actualidad son malezas de cultivo, y son utilizadas como verduras de hoja (Rapopport et al. 2009). Como plantea Capparelli (2006), considerando que las propiedades de las plantas en el presente son las mismas que tenían en el pasado, podemos suponer que sus aplicaciones o usos durante la ocupación del sitio fueron similares a las actuales en el área o en otras zonas del noroeste argentino. Siguiendo con este pensamiento podemos inferir que las mismas pudieron ser utilizadas quizá como verduras de hoja, descartando la parte reproductiva no utilizable en los fogones. Lo mismo se podría pensar para las vainas del Prosopis sp. o "algarroba" las cuales son utilizadas para la elaboración de jarabes, harinas, bebidas refrescantes y bebidas fermentadas (Capparelli 2007; Cutler y Cárdenas 1981: Fig. 1). Además, la madera de algarrobo proporciona un excelente combustible para hornos de metalurgia y cocción de ladrillos en la actualidad; la corteza y la raíz son usadas con fines tintóreos en la confección de tejidos en telar. En la medicina popular, varias especies de Prosopis sp. son utilizadas para curar afecciones de los ojos (Burkart 1952; FAO 2007).

Del Trichocereus sp. se consumen los frutos dulces, la madera se utiliza para construcciones rústicas, muebles y artesanías (Halloy 2008) y también se registra para la elaboración de "yista" utilizada en la salivación de hojas de coca en algunas comunidades de la puna (Fernández Distel 1984, 1997; Hilgert 2000). El maíz es consumido y preparado de manera variable. Los granos suelen comerse molidos, 
tostados, asados, hervidos o fermentados, o pueden ser servidos en diversos platos y bebidas como locro, humita, mazamorra, pochoclo, chicha, entre otros.

Del análisis de los esquemas de cuantificación utilizados encontramos que los taxa mejor representados en el sitio son Zea mays, Trichocereus sp. y Prosopis sp. Si asumimos que estos esquemas representan acceso, obtención y consumo, estas evidencias podrían indicar que el maíz habría sido localmente cultivado. Pudo sumarse al mismo, una importante fuente de recursos alimenticios silvestres. Además del Prosopis sp. y Trichocereus sp., los taxa silvestres más comunes en el sitio incluyen semillas de Opuntia sp., Portulaca sp. y Polygonum sp. La mayor parte de estos taxa silvestres, junto con el maíz, son hallazgos comunes en muchos sitios arqueológicos del NOA (Oliszewski et al. 2008). Esta diversidad de especies implica distintas formas de aprovisionamiento, relacionados con los diversos tipos de prácticas sociales que pudieran haber estado involucradas en la organización del trabajo necesario para su obtención o producción.

La distribución de restos en los recintos excavados no es homogénea, teniendo algunos mayores densidades que otros. Las mayores densidades fueron registradas en los estratos de los poblados (E3, E4, E5), pudiendo indicar que, en estos espacios, se realizaron tareas relacionadas con la manipulación de alimentos, ya sea procesamiento o consumo. De estos estratos sobresale el Recinto 161, con alta densidad de macrorrestos vegetales comestibles. Durante la excavación se definió un importante fogón y se recuperaron fragmentos de una olla culinaria que evidencian exposición al fuego. Se tomó una muestra para datación por radiocarbono que proporcionó una fecha que cae definidamente, en tiempos pre-inca [Años calibrados d. C. con $95.4 \%$ de probabilidad: 1229-1252 (4.7\%); 1259-1400 (90.7\%)]. El recinto es de buena factura y se asocia, espacialmente, a un conjunto de edificios del sector $X$ que se destacan por la imbricación de bloques líticos negros, rojizos y blancos, colores típicos del estilo Santamariano.

Del mismo modo, altas densidades de restos vegetales comestibles se dieron en los recintos 250, 221 y 274. Este último es una estructura rectangular pequeña ubicado en uno de los márgenes del poblado Sur. En superficie no ofrecía ninguna característica que llamara la atención por lo cual no habría sido excavado si no hubiera salido sorteado en el muestreo. Sin embargo, proporcionó el mayor número de macrorrestos marcando claramente la índole doméstica de dicho espacio. Se encontraba, además, anexo a una piedra de gran tamaño con morteros cilíndricos.

Precisamente varios tipos de artefactos de molienda, móviles e inmóviles, se hallaron restringidos a estos estratos pudiendo inferir que en esos espacios la molienda fue una práctica destacada por la gran abundancia de estos artefactos. Los taxa que más contribuyeron en la densidad de estos estratos son Prosopis sp. y Zea mays, y es conocido que a ambos se los procesa para la obtención de distintos productos alimenticios 
(Capparelli 2007a, 2007b). Esto apoyaría aún más la hipótesis que en estos estratos el procesamiento de alimentos fue una actividad importante.

El valor de densidad de E1 fue intermedio y el porcentaje de contribución de los taxa indica que las especies silvestres Trichocereus sp. y Prosopis sp. y la cultivada Zea mays son las que más aportaron a la densidad en este estrato. Analizando esto junto con las evidencias halladas durante las excavaciones, así como el análisis de la arquitectura permitirían suponer que en los recintos de ese estrato el consumo de vegetales alimenticios fue importante, pero no el procesamiento. En dicho estrato sobresalen los recintos residenciales 4 y 33, los cuales presentaron las mayores densidades de restos. Durante la excavación se pudo observar la presencia de espículas dispersas de carbón en el R4, y en el R33 se pudo determinar una estructura de combustión que provenía de un fogón en cubeta. Este edificio, ubicado en el Sector V o barrio Sudeste de la cumbre, es de excelente construcción, con lajas negras seleccionadas sobresaliendo sobre el espolón y disfrutando de una extraordinaria visibilidad del poblado bajo y de los Nevados del Aconquija.

Como ya destacamos, en este estrato no se han hallado artefactos de molienda, restos arqueobotánicos ni evidencias durante la excavación que pudieran sugerir cierto procesamiento de alimentos. De esta información se desprende una interesante consecuencia de índole social: que habrían existido sectores residenciales en el alto del cerro que recibían recursos alimenticios procesados por otros segmentos sociales, emplazados en los barrios del pedemonte. Estos datos refuerzan en forma independiente, la hipótesis planteada acerca de la existencia de diferencias sociales intrasitio (Tarragó 2011: 54).

En el estrato 2 sólo se recuperaron restos de Trichocereus sp. en uno de los recintos excavados (R 287). Una de las posibles explicaciones de la baja cantidad de hallazgos, así como de diversidad, es que en el sorteo de las cuadrículas a excavar dentro de los recintos no coincidió con las áreas de fogón que, normalmente, se encuentran cercanas a uno de los muros o en un ángulo. Otra posible explicación remitiría a la asignación de área ceremonial que se le dio a la Quebrada del Puma, pudiéndose pensar que en este estrato no se procesaban alimentos y tampoco se consumían comidas elaboradas en algunos de los edificios de propósitos especiales. En cambio, es muy probable el consumo de chicha cuyo procesamiento se habría realizado al pie, en el sector XIII de megalitos con numerosas oquedades de mortero. Como se ha planteado (Tarragó 2011; Tarragó y González 2004) los residentes de la cumbre (Estrato 1) y de la Quebrada del Puma (Estrato 2) requerían de los productos y de las materias primas que se producían en altitudes más bajas y alejadas del centro, y a partir de los macrorrestos, este planteo se podría extender tanto para la obtención como para el procesamiento de vegetales comestibles. 
En relación a los macrorrestos recuperados correspondientes a Zea mays, los fragmentos de marlos (ya sean porciones de los mismos o cúpulas sueltas) están más densamente concentrados que los granos, en los estratos 4 y 5 , observándose una notable diferencia con lo que sucede el estrato 1 donde los granos predominan sobre los marlos. La presencia de marlos o cúpulas indicaría que, en mayor o menor intensidad, hubo procesamiento de este cereal en el área residencial de Rincón Chico 1, tal como lo plantean algunos autores (Lennstrom 1992; Miller 1988) a partir de este tipo de evidencia. Sobre esto también podemos inferir que este taxón fue incorporado en los recintos sin desgranar o que los residentes tuvieron un cuidado especial con los granos (Lennstrom 1992). La baja o nula proporción de granos sugiere una cierta funcionalidad diferencial de los recintos en relación al procesamiento y/o consumo del maíz y rutas diferentes para granos y marlos, una vez efectuado el desgranado en el sitio. Los primeros debieron ser convertidos principalmente en harina y no tener contacto directo con el fuego mientras que los marlos siguieron circulando en el sitio como combustible para los fogones interiores. Siguiendo estas ideas, se puede pensar que en los estratos del poblado del pedemonte se realizaba el procesamiento de este cereal, por la presencia de una mayor abundancia de granos, que durante su preparación hayan caído accidentalmente al fuego o que hayan sido descartados por daños.

En cuanto al consumo de variedades de maíz en Rincón Chico, cabe mencionar la aproximación preliminar realizada por Raffaele, bajo el asesoramiento del Ing. Cámara Hernández, sobre los macrorrestos carbonizados de marlos del sitio 15. En ese sitio se colectaron en forma directa de varios fogones, 25 restos de marlos casi completos. Los mismos presentaron afinidad morfológica con las razas actuales "perla", "pisingallo", "chulpi" y "capia", dándose la frecuencia relativa mayor en esta última (Cámara Hernández y Miante Alzogaray 1989; Raffaele 2008). En sitios arqueológicos de Antofagasta de la Sierra, en la Puna Salada, donde la preservación de restos es excelente, se ha podido realizar un estudio pormenorizado de las variedades presentes guardando correspondencia con las aquí mencionadas (Babot 2006; Rodríguez 2013: 327).

\section{Conclusiones}

La baja densidad y diversidad de restos recuperados en el sitio RCh1 se pueden relacionar con los factores de preservación tanto natural como cultural, así como con las técnicas de excavación y recuperación de los restos. Por otro lado, es indudable que los especímenes encontrados en los sitios son solo una fracción de la totalidad de las plantas que pudieron utilizar los habitantes del asentamiento.

La distribución de carporrestos en el sitio RCh1 sugiere que la preparación de comidas ocurría en lugares específicos. Se encontró evidencia de esta actividad en los estratos pertenecientes al poblado bajo (E3, E4 y E5), donde se obtuvo la mayor densidad y variabilidad de restos arqueobotánicos, así como la concentración de cúpulas de maíz 
y la distribución restringida de los artefactos de molienda. En los otros estratos de las partes altas del Cerro de Rincón Chico (E1 y E2) la baja densidad de hallazgos pudo estar influenciada por la cantidad de recintos excavados (E2) y por las prácticas de limpieza de las superficies de ocupación de los recintos. El tipo de restos recuperados no estarían indicando que en esos espacios el procesamiento haya sido una práctica importante, y se podría pensar que los residentes de estos estratos necesitaban de los productos elaborados que producían los habitantes de la altitudes bajas.

Los restos de vegetales silvestres, el algarrobo, el cardón y Opuntia sp., son especies que se encuentran fácilmente en las inmediaciones del sitio y poseen una larga tradición de uso por los habitantes del Noroeste argentino. Estas especies estarían indicando que la recolección fue una práctica importante y esto podría señalar una organización del trabajo necesaria para su obtención.

Con respecto a las especies cultivadas se encontraron evidencias fehacientes tan solo de maíz. Pero, como se discutió anteriormente, diversos factores influyen en la preservación de los restos y la evidencia negativa no necesariamente implica que un recurso haya sido dejado de lado.

Este análisis se suma a la primera experiencia efectuada por Arriaga et al. (1994). Aunque en una baja densidad en comparación con otros sitios del NOA, brinda datos importantes para la observación de la variabilidad intra-sitio y las particularidades que se dan en los diversos sectores de vivienda. Por otro lado, muestra la importancia de la excavación por muestreo de las estructuras de vivienda en los grandes asentamientos de los valles, aspecto muy poco contemplado hasta el presente. En tercer lugar pone de manifiesto el indiscutible valor de la técnica de flotación para la obtención restos botánicos carbonizados que conserven atributos que posibiliten su identificación. En ese sentido destacamos la necesidad de su utilización en forma sistemática, en toda clase de excavaciones llevadas a cabo en áreas de viviendas arqueológicas.

Como punto final deseamos expresar nuestro propósito de continuar los estudios de macrorrestos incorporando los otros sitios de la localidad de Rincón Chico y de otros sitios del valle de Santa María y aledaños. Estos estudios permitirán hacer una comparación más amplia en el espacio y en el tiempo, de las cadenas de procesamiento, uso y consumo de vegetales en épocas prehispánicas.

Agradecimientos: A la Prof. Susana F. Renard, sin cuyo cuidadoso trabajo al implementar la flotación manual, no hubiera sido posible esta investigación. A la Dra. Mirta Arriaga del Museo Argentino de Ciencias Naturales que aceptó dirigir el primer estudio de la flotación en el recinto 218. A las Dras. Verónica Lema y María Lelia Pochettino, del Museo de Ciencias Naturales (Universidad Nacional de La Plata), por el apoyo brindado, la orientación en la identificación de restos y el acceso a los aparatos necesarios para el análisis. Del mismo modo, nuestro profundo agradecimiento a todos los integrantes de las campañas de campo de 1992 y 1995 que llevaron adelante un intenso y arduo trabajo. 


\section{Notas}

${ }^{1}$ Los trabajos fueron financiados, entre otros, por ANPCyT-PICT 34511, PICT 201000113; Beca inicial en el marco de este proyecto desde el 1-7-2012 a 1-7- 2015. UBACYT (2011-2014) 20020100100632.

2 Lamentamos que algunas muestras de flotación del estrato 3, Sector VIII, no pudieron ser analizadas por no ubicarse en el depósito.

\section{Bibliografía citada}

Arriaga, M.; Renard, S. y S. Aliscioni

1994 La recuperación de microespecímenes en la excavación arqueológica de Rincón Chico 1. Identificación de restos botánicos. Actas XI Congreso Nacional de Arqueología Argentina. Revista del Museo de Historia Natural de San Rafael 29 (1/4), 17 Parte: 7-17.

Babot, M.

2006 El papel de la molienda en la transición hacia la producción agropastoril: un análisis desde la Puna Meridional argentina. Estudios Atacameños 32: 75-92.

Burkart, A.

1952 Las Leguminosas Argentinas Silvestres y Cultivadas. ACME Editores, Buenos Aires.

Cabrera, A.

1976 Regiones fitogeográficas argentinas. En Enciclopedia Argentina de Agricultura y Jardinería, T. 2(1). ACME Editores, Buenos Aires.

Cabrera, N.

1990 Estudio sobre la vegetación natural en el área arqueológica de Rincón Chico, Valle de Santa María (Catamarca). Informe MS, 17 pp. Museo Etnográfico, Buenos Aires.

Cámara Hernández, J. y A. Miante Alzogaray

1989 Maíz (Zea mays, ssp. mays) arqueológico del Pucará de Tilcara, Jujuy, Argentina. Revista de la Facultad de Agronomía 10(3): 99-108.

Capparelli, A.

2007a Los productos alimenticios derivados de Prosopis chilensis (Mol.) Stuntz y P. flexuosa DC., Fabaceae, en la vida cotidiana de los habitantes del NOA y su paralelismo con el algarrobo europeo. Kurtziana 33: 1-19.

2007b El Algarrobo blanco y negro -Prosopis chilensis (Mol.) Stuntz y P. flexuosa DC, Fabaceae, en la vida cotidiana de los habitantes del NOA: subproductos alimenticios. Kurtziana 33: 103-119. 
Capparelli, A.

2008 Caracterización cuantitativa de productos intermedios y residuos derivados de alimentos del algarrobo (Prosopis flexuosa y P. chilensis, Fabaceae): aproximación experimental aplicada a restos arqueobotánicos desecados. Darwiniana 46(2): 175- 201.

Cutler, H. y M. Cárdenas

1981 Chicha, una cerveza sudamericana indígena. En La Ttecnología en el Mundo Andino, H. Lechtman y A. Soldi (eds.), pp. 247-260. Universidad Nacional Autónoma, México.

FAO (Food and Agriculture Organization)

2007 El género Prosopis "algarrobo" en América Latina y el Caribe. Distribución, bioecoloía, usos y manejo. www.Fao.org/docrep/006/ad314s/AD314S01.htm. Consultado el 11 de abril 2015.

Fernandez Distel, A.

1984 Contemporary and archaeological evidence of llipta elaboration from cactus Trichocereus pasacana in Northwest Argentina. En Social and Economic Organization in the Prehispanic Andes, D. Browman, R. Burger y A. Rivera (eds.), pp. 193-203. BAR International Series 194, Oxford.

1997 La yista del cardón pasacana (Trichocereus pasacana (Web.) Britton et Rose Cactacea) en la Provincia de Jujuy, Argentina. Parodiana 10: 1-9.

González, L.

1995 Recursos y organización de la producción metalúrgica prehispánica en la región Centro-Sur. Un caso de estudio. Actas del XIII Congreso Nacional de Arqueología Chilena, Hombre y Desierto 9: 213-224. Antofagasta.

2010 Fuegos sagrados. El taller metalúrgico del sitio 15 de Rincón Chico (Catamarca, Argentina). Boletín del Museo Chileno de Arte Precolombino 15(1): 47-62.

Grondona, M.

1978 Observaciones fitogeográficas en la provincia de Catamarca. En Geografía de Catamarca GAEA, pp. 107-135. Sociedad Argentina de Estudios Geográficos, Buenos Aires.

Halloy, S.

2008 Crecimiento exponencial y supervivencia del cardón (Echinopsis atacamensis subsp. pasacana) en su límite altitudinal (Tucumán, Argentina). Ecología en Bolivia 43(1): 6-15. 
Hilgert, N.

2000 Especies vegetales utilizadas en la insalivación de hojas de coca (Erythroxylum coca var. coca, Erythroxylaceae). Darwiniana 38(3-4): 241-252.

Lennstrom, $\mathrm{H}$.

1992 Botanical remains from the Calchaqui Archaeological Project 1990. Archaeobotany Laboratory Report 29. University of Minnesota.

Lennstrom, H. y C. Hastorf

1992 Testing old wives tale's in Paleoethnobotany: a comparison of bulk and scatter sampling schemes from Pacán, Perú. Journal of Archaeological Science 19: 205-229.

1995 Interpretation in context: sampling and analysis in Paleoethnobotany. American Antiquity 60(4): 701-721.

Miller, N.

1988 Ratios in Paleoethnobotanical analysis. En Current Paleoetnobotany. Analytical Methods and Cultural Interpretations of Archaeological Plant Remains, C. Hastorf y V. Popper (eds.), pp. 72-85. University of Chicago, Chicago.

Mueller, J.

1979 Sampling in Archaeology. The University of Arizona Press, Tucson.

Munson, P., P. Parmalee y R. Yarnell

1971 Subsistence ecology of Scovill, a terminal Middle Woodland village. American Antiquity 36: 410-431.

Oliszewski, N., J. Martínez y M. Caria

2008 Ocupaciones prehispánicas en una quebrada de altura: el caso de la Cueva de Los Corrales 1 (El Infiernillo, Tafí del Valle, Tucumán). Relaciones de la Sociedad Argentina de Antropología XXXIII: 209-221.

Pearsall, D.

1988 Interpreting the meaning of macroremain abundance: the impact of source and context. En Current Paleoetnobotany. Analytical Methods and Cultural Interpretations of Archaeological Plant Remains, C. Hastorf y V. Popper (eds.), pp. 97-118. University of Chicago, Chicago.

Pochettino, M. L. y M. C. Scattolin

1991 Identificación y significado de frutos y semillas carbonizadas de sitios arqueológicos formativos de la ladera occidental del Aconquija (Catamarca, República Argentina). Revista del Museo de La Plata N. S. t. 9, Antropología 71: 169-181. 
Popper, V.

1988 Selecting quantitative measurements in Paleoetnobotany. En Current Paleoetnobotany. Analytical Methods and Cultural Interpretations of Archaeological Plant Remains, C. Hastorf y V. Popper (eds.), pp. 53-71. University of Chicago, Chicago.

Raffaele, L.

2008 Estudio paleoetnobotánico sobre variabilidad de maíz en el valle de Yocavil. En Estudios Arqueológicos en Yocavil, M. Tarragó y L. González (eds.), pp. 277-321. Buenos Aires.

Ruiz Huidobro, O.

1972 Descripción geológica de la hoja 11e, Santa María. Boletín No 134. Ministerio de Industria y Minería, Subsecretaría de Minería, Servicio Nacional Minero Geológico, Buenos Aires.

Tarragó, M.

1987 Sociedad y sistema de asentamiento en Yocavil. Cuadernos del Instituto Nacional de Antropología 12: 179-196.

1995 Desarrollo regional en Yocavil: una estrategia de investigación. Actas del XIII Congreso Nacional de Arqueología Chilena, Hombre y Desierto 9: 225-236. Antofagasta.

2007 Ámbitos domésticos y de producción artesanal en el Noroeste argentino prehispánico. Intersecciones en Antropología 8: 87-100.

2011 Poblados tipo pukara en Yocavil El plano de Rincón Chico 1 (Catamarca, Argentina). Estudios Sociales del NOA 11: 33-61.

Tarragó, M. y L. González.

2004 Arquitectura social y ceremonial en Yocavil, Catamarca. Relaciones de la Sociedad Argentina de Antropología XXIX: 297-316.

Tarragó, M.; Renard, S. y L. González

1992 Proyecto arqueológico Valle de Yocavil. Informe campaña 1992. Palimpsesto 2: 133138.

Winton, A. y K. Winton

1932 The Structure and Composition of Foods (Cereals, Starch, Oil Seeds, Nuts, Oils, Forage Plants). Vol. 1. John Willey \& Sons, New York.

1935 The Structure and Composition of Foods. Vol. 2. John Willey \& Sons, New York. 